\title{
New mouse model reveals nitrosative stress as a novel driver of HFpEF
}

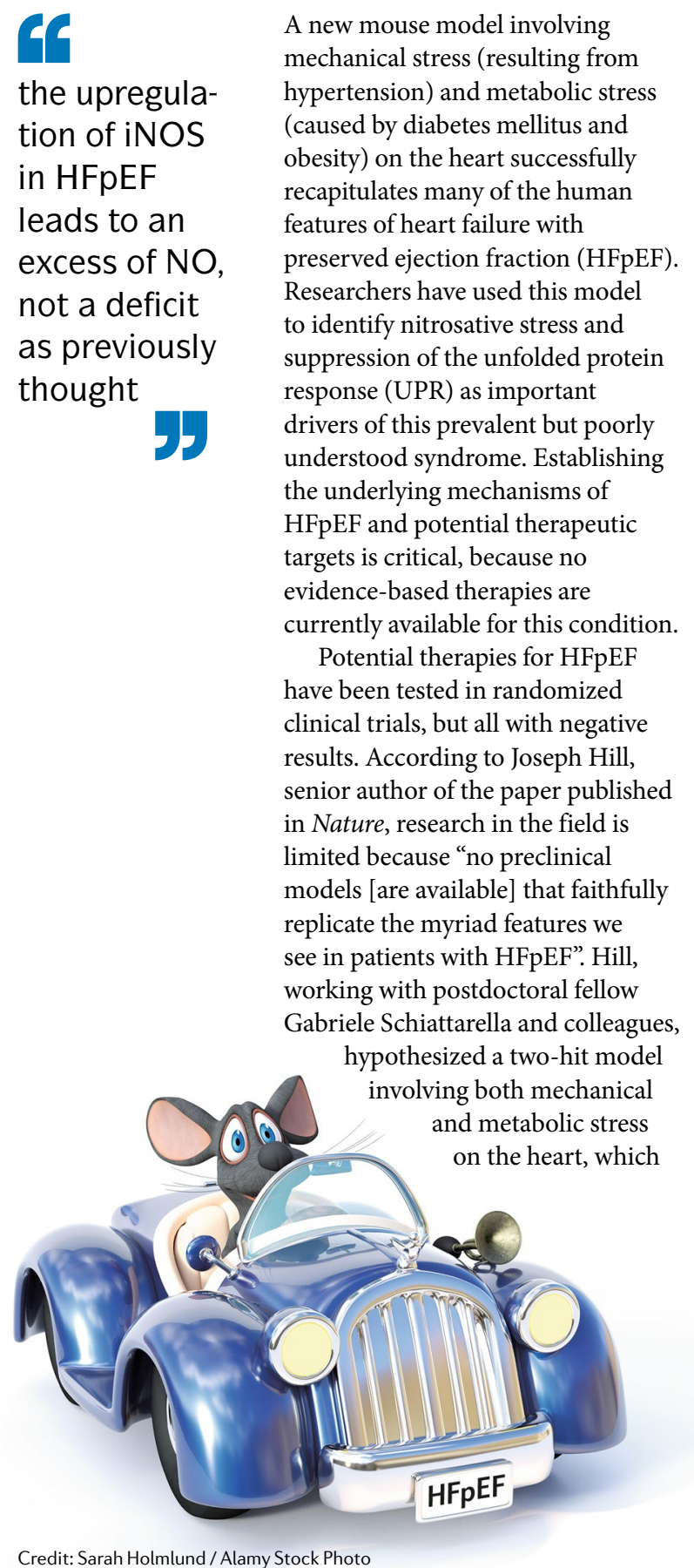

recapitulates the hypertension, obesity and diabetes that typically coexist in patients with HFpEF.

Mice were exposed to one of four regimens: a high-fat diet, drinking water containing $N^{\omega}$-nitroL-arginine methyl ester (L-NAME; an inhibitor of constitutive nitric oxide synthases), a combination of both (the two-hit model) or a standard chow diet. Although some features of HFpEF were observed in the animals fed a high-fat diet and in those receiving L-NAME, mice that received the combined high-fat and L-NAME diet developed a phenotype most closely resembling that of human HFpEF, including cardiac hypertrophy, pulmonary congestion, exercise intolerance and worsened diastolic function. "This new model recapitulates the realities [of $\mathrm{HFpEF}$ ] seen in patients more than other models we have seen in the literature," states Hill.

Using this new mouse model, the researchers investigated the underlying mechanisms of HFpEF and established that the UPR was downregulated in mice fed a high-fat plus L-NAME diet, a finding that was confirmed in myocardial tissue from patients with HFpEF. The UPR is an evolutionarily conserved adaptive response that can mitigate stress in conditions that disrupt protein quality control mechanisms. Further experiments revealed a state of 'meta-inflammation' (inflammation stemming from metabolic toxicity seen in obesity and diabetes). Robust upregulation of inducible nitric oxide synthase (iNOS or NOS2) produces a surfeit of circulating nitric oxide (NO). This NO covalently binds to cysteine thiol groups in many proteins (a process termed $S$-nitrosylation), altering their structure and function. Of note, $S$-nitrosylation of the endonuclease inositol-requiring protein $1 \alpha$ (IRE1 $\alpha)$ reduces the activity of this protein and leads to defective splicing of mRNA encoding X-box-binding protein 1 (XBP1). Spliced XBP1 is a transcription factor that activates genes encoding proteins involved in the UPR. Indeed, the IRE1 $\alpha-\mathrm{XBP} 1$ axis is the most evolutionarily conserved branch of the UPR. Importantly, suppression of iNOS (either pharmacologically with $\mathrm{L}-\mathrm{N}^{6}$-(1-iminoethyl) lysine (L-NIL) or genetically) or cardiomyocyte-restricted overexpression of spliced XBP1 improved - but did not completely normalize - the HFpEF phenotype.

Some of the clinical trials in HFpEF have tested the use of NO donors such as the phosphodiesterase 5 inhibitor sildenafil; Hill believes that their study explains why these trials have been unsuccessful: "the upregulation of iNOS in HFpEF leads to an excess of NO, not a deficit as previously thought". The researchers are now working to develop a nonhuman primate model of HFpEF. If successful, they plan to target iNOS, as they did in mice. "If iNOS inhibition is beneficial, then I envision moving rapidly towards a first-in-human clinical trial," predicts Hill.

Gregory B. Lim

ORIGINAL ARTICLE Schiattarella, G. G. et al. Nitrosative stress drives heart failure with preserved ejection fraction. Nature $\mathbf{5 6 8}, \mathbf{3 5 1 - 3 5 6}$ (2019)

FURTHER READING Dunlay, S. M. et al. Epidemiology of heart failure with preserved ejection fraction. Nat. Rev. Cardiol. 14, 591-602 (2017)|Borlaug, B. A. The pathophysiology of heart failure with preserved ejection fraction. Nat. Rev. Cardiol. 11, 507-515 (2014) 